\title{
28
}

\section{Assessment of performance and optimal strategies for inspection and maintenance of concrete structures using reliability based expert systems}

P. Thoft-Christensen

CSR, P.O.Box 218, DK-9100 Aalborg, Denmark

\section{SUMMARY}

Expert systems for optimal reliability-based inspection and maintenance of reinforced concrete bridges are described. The deterioration is corrosion of the reinforcement due to carbonation and chloride. Expert system module BRIDGE1 is used at the bridge site to assist during the inspection. BRIDGE2 is used after an inspection during the detailed analysis of the bridge when testing in the laboratory has taken place.

\section{INTRODUCTION}

The main objective of the project is to optimise strategies for inspection, maintenance and repair of reinforced concrete bridges by developing improved methods for modelling the deterioration of existing as well as future structures using reliability based methods and expert systems are presented with special emphasis on reliability assessment, updating, and the software modules. Further, the functionalities and the structure of the expert systems and the optimal strategy is briefly discussed. The paper is based on Thoft-Christensen [8].

\section{MODELLING OF THE DETERIORATION}

Only one type of deterioration is included namely corrosion of the reinforcement due to carbonation and chloride. The area of the reinforcement $A(t)$ as function of time $t$, the depth of the carbonation front $d_{c}$, and the chloride concentration $\mathrm{C}$ are estimated on basis of well known techniques, see Thoft-Christensen [7]. It is assumed that the area $A(t)$ of the $n$ reinforcement bars in a cross section as function of time $t$ can be determined by

$$
A(t)=\left\{\begin{array}{cl}
n D_{i}^{2} \pi / 4 & \text { for } t \leq T_{i} \\
n(D(t))^{2} \pi / 4 & \text { for } T_{i}<t \leq T_{i} \\
0 & \text { for } t>T_{i}
\end{array}\right.
$$

where $T=T_{i}+D_{i} /\left(0.023 i_{\text {corr }}\right)$. The diameter $\mathrm{D}(\mathrm{t})$ of a single bar (in $\mathrm{mm}$ ) at time $\mathrm{t}$ is modelled by $\mathrm{D}(\mathrm{t})=D_{i}-0.023\left(\mathrm{t}-T_{i}\right) i_{\text {corr }} . D_{i}$ is the initial diameter of a single bar, and $i_{\text {corr }}$ is the rate of corrosion in terms of a mean corrosion current density in $\mu \mathrm{A} / \mathrm{cm}^{2}$. It is assumed that a corrosion current of $1 \mathrm{~A} / \mathrm{cm}^{2}$ is equivalent to an average oxidation or dissolution of approximately $1.6 \mathrm{~mm} /$ year from the surface of steel. The corrosion initiation 
time $T_{i}$ (in years), due to carbonation (as function of the carbonation front $d_{c}$ in $\mathrm{mm}$ ) and due to chloride (as function of the chloride diffusion coefficient $D_{c}$ in $\mathrm{cm}^{2} / \mathrm{sec}$ ) is estimated by:

carbonation: $T_{i}=\left(\frac{d}{K}\right)^{2} \quad$ chloride: $\quad T_{i}=\frac{d^{2}}{4 D_{c}}\left(\operatorname{erf}^{-1}\left(\frac{C_{c r}-C_{0}}{C_{i}-C_{0}}\right)\right)^{-2}$

where $\mathrm{d}$ is the concrete cover in $\mathrm{cm}, \mathrm{K}$ is a constant depending of the concrete cover depth $\mathrm{d}$, concrete mix proportions, water/cement ratio, cement content etc. $C_{0}$ is the equilibrium chloride concentration (in \% by weight of cement) on the concrete surface and $C_{c r}$ is the critical chloride concentration at the reinforcement level. Using Fick's law of diffusion the chloride concentration $\mathrm{C}(\mathrm{x}, \mathrm{t})$ at depth $\mathrm{x}$ from the surface and at time $\mathrm{t}$ can be determined by

$C(x, t)=C_{0}\left(1-\operatorname{erf}\left(\frac{x}{2 \sqrt{D_{C} t}}\right)\right.$

Table 1

Definition of stochastic variables

\begin{tabular}{lll}
\hline & Stochastic variable & Distribution type \\
\hline $\mathrm{X}_{1}$ & Concrete cover & Normal \\
$\mathrm{X}_{2}$ & Height of beam & Normal \\
$\mathrm{X}_{3}$ & Height of deck & Normal \\
$\mathrm{X}_{4}$ & Initial diameter of reinforcement & Normal \\
$\mathrm{X}_{5}$ & With of column & Normal \\
$\mathrm{X}_{6}$ & Dept of column & Normal \\
$\mathrm{X}_{7}$ & Compression yield stress, concrete & Normal \\
$\mathrm{X}_{8}$ & Yield stress of reinforcement & Normal \\
$\mathrm{X}_{9}$ & Uniformly distributed dead load & Normal \\
$\mathrm{X}_{10}$ & Uniformly distributed traffic load & Gumbel \\
$\mathrm{X}_{11}$ & Point traffic load & Gumbel \\
$\mathrm{X}_{12}$ & Chloride concentration on concrete surface & Normal \\
$\mathrm{X}_{13}$ & Chloride diffusion coefficient & Lognormal \\
$\mathrm{X}_{14}$ & Coefficient rate of carbonation & Normal \\
$\mathrm{X}_{15}$ & Rate of corrosion & Normal \\
$\mathrm{X}_{16}$ & Measurement uncertainties & Normal \\
\hline
\end{tabular}

\section{RELIABILITY ASSESSMENT}

The reliability of the bridge is measured using the reliability index $\beta$ for a single failure element or for the structural system (the bridge) (Thoft-Christensen \& Baker [5], Thoft-Christensen \& Morutsu [6]). The overall requirement is that the expected reliability index should never be smaller than some minimum reliability index. In the present version of 
the expert systems 3 failure modes are implemented namely: "positive" and "negative" bending failure of a reinforced T-beam (see figure 1a) and compression failure of a rectangular reinforced column ( two models, namely with and without bending moments, see figure $1 b$ ). The probability of failure is estimated using the software package RELIAB.

a)

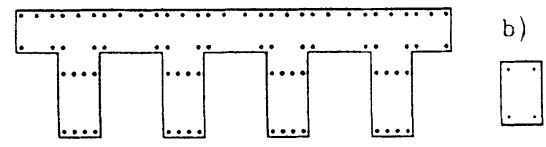

Figure 1. a) reinforced T-beam; b) rectangular reinforced column.

\section{FAILURE PROBABILITY UPDATING}

Two main types of updating of the probability of failure estimates are considered:

- Updating of stochastic variables based on measured samples of the stochastic variables,

e.g. measurements of the yield strength of the reinforcement.

- Updating based on general information, e.g. the observation that the structure is not

failed or that a corrosion degree is smaller than a certain value is measured.

Basic variable updating is performed within the framework of Bayesian statistical theory (Lindley [2], Aitchison \& Dunsmore [1]). The updating based on general information is mainly based on the Bayesian methods suggested by Madsen [3] and Rackwitz \& Schrupp [4].

Let the density function of a stochastic variable $X$ be given by $f_{X}(x, \Theta)$, where $\Theta$ are parameters defining the distribution of $X$ The parameters $\Theta$ are treated as uncertain parameters (stochastic variables). $f_{X}(x, \Theta)$ is therefore a conditional density function $f_{X}(x \mid \Theta)$. The initial (or prior) density function for $\Theta$ is called $g_{\Theta}^{\prime}(\theta)$

When an inspection is performed $\mathrm{n}$ realisations $\vec{x}=\left(x_{1}, \ldots, x_{n}\right)$ of the stochastic variable $X$ are obtained. The inspection results are assumed to be independent. An updated density function $\Theta$ taking into account the inspection results is then defined by

$g_{\Theta}^{\prime \prime}\left(\theta \mid \bar{x}^{*}\right)=\frac{f\left(\bar{x}^{*} \mid \theta\right) g_{\Theta}^{\prime}(\theta)}{\int f_{n}(\bar{x} \mid \theta) g_{\Theta}^{\prime}(\theta) d \theta} \quad, \quad f_{X}\left(x \mid \bar{x}^{*}\right)=\prod_{i=1}^{n} f_{X}\left(x_{i} \mid \theta\right)$

The updated density function of $X$ taking into account the realisations $\vec{x}^{*}$ is then obtained by

$f_{X}\left(x \mid \bar{x}^{*}\right)=\int f_{X}(x \mid \theta) g_{\Theta}^{\prime \prime}\left(\theta \mid \vec{x}^{*}\right) d(\theta)$

In the expert systems the functions $g_{\Theta}^{\prime}(\theta), g_{\Theta}^{\prime \prime}(\theta)$, and $f_{X}\left(x \mid \bar{x}^{*}\right)$ are implemented for normal distributions with unknown mean and standard deviations, for the Gumbel distribution with unknown location parameter, for the Weibull distribution with unknown location parameter, for the Frechet distribution with unknown location parameter, and for the exponential distribution with unknown location parameter. 


\section{THE EXPERT SYSTEM FUNCTIONALITIES}

The expert system is divided into two expert system modules which are used in two different situations, namely by the inspector of the bridge during the inspection at the site of the bridge and after the inspector has returned to his office. During the inspection the expert system will supply information on: the causes of observed defects, appropriate diagnosis methods, and related defects. Further the inspector will be asked to record the inspection results so that they can be used later for e.g. assessment of the reliability of the bridge and in the decision whether a detailed structural assessment is needed.

A detailed analysis of the state of the bridge after an inspection takes place when the inspector has returned to his office and after testing in the laboratory has taken place. The output of the analysis includes an updated estimation of the reliability of the bridge, decision whether a structural assessment should take place, decision whether repair should take place, relevant repair procedures, and the time for repair. Expert knowledge is used to improve the quality of the decisions.

\section{ARCHITECTURE OF THE EXPERT SYSTEMS}

The architecture of the expert system is shown in figure 2. It consists of two main modules called BRIDGE1 and BRIDGE2. The BRIDGE1 expert system is used at the site of the bridge. BRIDGE2 is used by the engineer in the office.

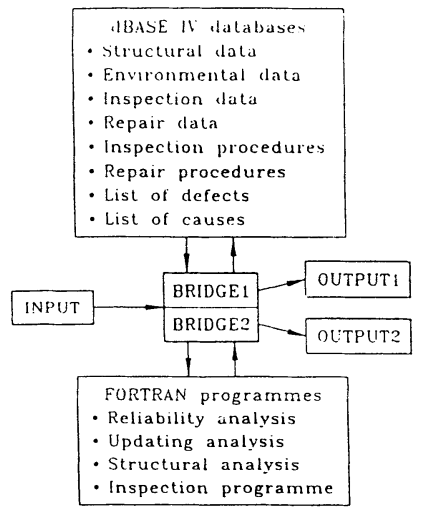

Figure 2. Architecture of the expert systems.

\section{APPLICATION OF THE EXPERT SYSTEMS}

The general inspection, maintenance, and repair model from inspection no. $\mathrm{i}$ at time $t_{i}$ to inspection no. $\mathrm{i}+1$ at time $t_{i+1}=t_{i}+\Delta t$ is indicated in figure 3 , where also the application

of the modules BRIDGE1 and BRIDGE2 is shown. C, D and A are current, detailed inspections and structural assessments respectively. $\mathrm{M}$ is maintenance and repair of minor defects and R is Structural repair. B1 indicates application of BRIDGE1. B2(M), B2(I) and $\mathrm{B} 2(\mathrm{R})$ are the maintenance subsystem, the inspection module and the repair subsystem in $B R I D G E 2$ respectively. 


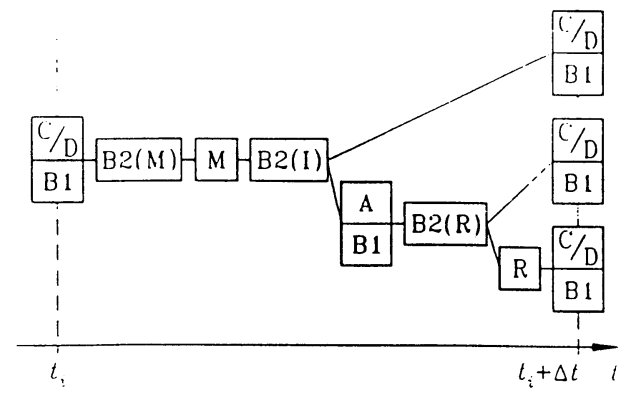

Figure 3. The inspection, maintenance, and repair model.

After a current or a detailed inspection BRIDGE2 is used to rate the maintenance and minor repair work needed and to decide if a structural assessment has to be performed. The decision is based partly on estimates of the reliability of the bridge and partly on expert knowledge. The decision does not include economic considerations.

After a structural assessment BRIDGE2 is used to decide if a repair has to be performed and also to give the optimal point of time for the repair. Expert knowledge as well as numerical algorithms are used. The decisions are partly based on a cost-based optimisation where different repair possibilities (selected by expert knowledge) and no repair are compared. The total expected costs are minimised using the FORTRAN inspection module.

\section{DECISION MODEL WITH REGARD TO STRUCTURAL ASSESSMENT}

Let $t_{i}$ be the time of a periodic inspection and let the updated reliability index at time $\mathrm{t}$ be $\beta\left(t, t_{i}\right)$. The general decision model with regard to the structural assessment can then be formulated as:

- If $\beta\left(t_{i+1}, t_{i}\right)>\beta^{\min }$ then the inspection at time $t_{i+1}$ should be a current or detailed inspection unless the damage is so serious that a structural assessment is needed. This decision is based on expert knowledge. $\beta^{\text {min }}$ is the minimum acceptable reliability index (e.g. 3.72).

- If $\beta\left(t_{i+1}, t_{i}\right) \leq \beta^{\min }$ then a structural assessment should be performed before the next periodic inspection

\section{MODELLING OF REPAIR}

After a structural assessment it must be decided whether the bridge should be repaired and if so, how the repair is performed. In order to decide which repair type is optimal after a structural assessment, the following optimisation problem is considered for each repair technique: 
$\max _{T_{R}, N_{R}} W\left(T_{R}, N_{R}\right)=B\left(T_{R}, N_{R}\right)-C_{R}\left(T_{R}, N_{R}\right)-C_{F}\left(T_{R}, N_{R}\right)$

s.t. $\quad \beta^{L}\left(T_{L}, T_{R}, N_{R}\right) \geq \beta^{\min }$

where the optimisation variables are the expected number of repair $N_{R}$ in the remaining lifetime and the time $T_{R}$ of the first repair. $\mathrm{W}$ is the total expected benefits minus costs in the remaining lifetime of the bridge. $C_{R}$ is the repair cost capitalised to time $\mathrm{t}=0$ in the remaining lifetime of the bridge. $C_{F}$ is the expected failure costs capitalised to time $\mathrm{t}=0$ in the remaining lifetime of the bridge. $T_{L}$ is the expected lifetime of the bridge. $\beta^{U}$ is the updated reliability index. $\beta^{\min }$ is the minimum reliability index for the bridge ( related to a critical element or to the total system). The repair decision is then based on the results of solving this optimisation problem but also on expert knowledge.

\section{BRIDGE1}

The expert system module BRIDGE1 contains useful information concerning the bridge being inspected and the defects being observed. The information includes: general information about the bridge, appropriate diagnosis methods for each defect, probable causes for each defect, and other defects related to a defect. It is also possible to create a provisional defect report. The general information about the bridge stored in the database for the selected bridge can be reviewed. The database contains information about: bridge site, design, budget, traffic, strength, load, deterioration, factors that models costs, and the cross-sections entered for the bridge. New cross-sections can be entered for the selected bridge. The information stored in the database for each cross-section contains: cross-section identification, geometry of crosssection (detailed description of the reinforcement layers for cross-sections in the deck), failure mode, and load data. Technical support can be provided for a defect. The technical support include:

- $\quad$ List of diagnosis methods that can be used to observe a selected defect

- List of probable causes of a selected defect.

- List of defects associated with the selected defect.

\section{BRIDGE2}

The expert system module BRIDGE2 is used to make a detailed analysis of the bridge after an inspection when testing in the laboratory has taken place. New bridges and cross-sections can be entered in the database and existing bridges and cross-sections can be edited. For the bridges in the database the following options are available: review provisional defect reports, enter inspection results, estimate the reliability index, plan maintenance work and estimate costs, plan structural repair work and estimate costs, and review the agenda of inspection for one bridge or all bridges. Further, the database can be updated after repair.

New bridges can be entered and existing bridges can be edited. The general information about the bridges stored in the database contains information about: bridge site, design, budget, traffic, strength, load, deterioration, factors that model the costs, and the cross-sections entered for each bridge. 
The reliability index for the bridge can be estimated by the integrated FORTRAN program RELIAB. Both the reliability index when no inspection results are taken into account and the updated reliability index when all inspections performed for the bridge are taken into account can be estimated.

The following submodules are integrated in BRIDGE2:

- $\quad$ BRIDGE2(M) is the maintenance/small repair submodule. This submodule is always used after a current or detailed inspection. It assists in selecting the maintenance work and repair of minor structural defects to be performed and estimate the maintenance costs. The defects are rated based on the defect classification in terms of rehabilitation urgency, importance of the structure's stability, and affected traffic recorded during the inspection .

- $\quad$ BRIDGE2(I) is the inspection strategy submodule. This submodule is always used after a current or detailed inspection. It assists in the decision whether a structural assessment is needed before the next periodic inspection. The decision taken in BRIDGE2(I) is mainly based on the updated reliability index for the bridge calculated by RELIAB (see figure 8). If the value of the updated reliability index for the bridge is acceptable then each of the defects detected at the latest periodic inspection and the combination of defects are investigated. Based on expert knowledge it is investigated whether from a structural point of view a defect or combinations of defects require a structural assessment.

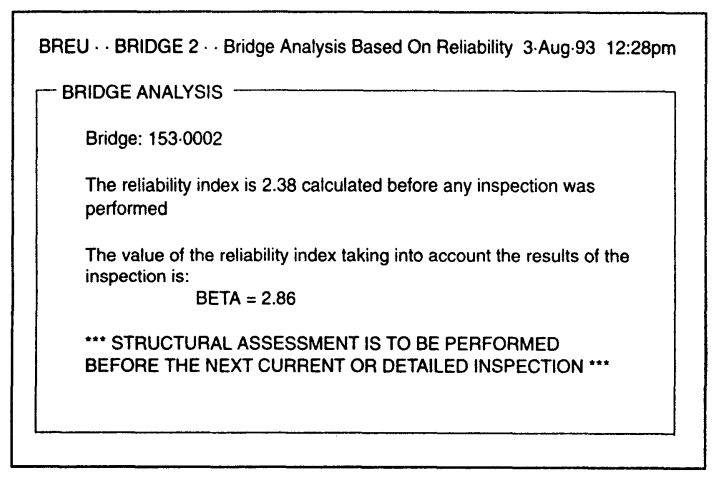

Figure 4. Decision tool related to structural assessment.

- $\quad$ BRIDGE2(R) is the repair submodule. This submodule is always used after a structural assessment. It assists in selecting the optimal structural repair technique (including no repair) to be performed, when the repair should be performed, and the number of repairs in the remaining lifetime of the bridge. Further, the expected benefits minus costs are estimated. The repair plan is optimised based on a cost-benefit analysis by the FORTRAN program INSPEC (see figure 5).

The FORTRAN program RELIAB can be used to estimate the reliability of a reinforced concrete bridge. Two different failure modes are considered, namely bending failure of the main beam of a bridge and compression failure of a column.

The FORTRAN program INSPEC can be used to estimate the optimal repair time and number of repairs for a given repair method. The estimation is based on a cost-benefit analysis 
for the bridge. The total expected benefits minus expected repair and failure costs in the remaining lifetime $\mathrm{of}$ the bridge is optimised.

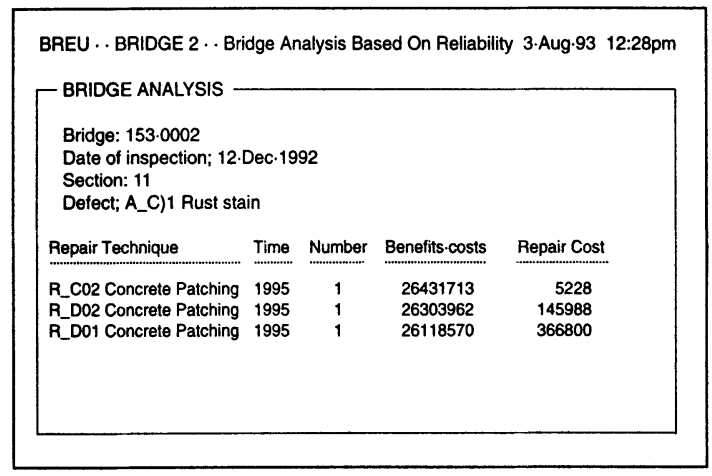

Figure 5. Optimised repair plan for the defect 'rust stain'

\section{ACKNOWLEDGEMENT}

This paper presents results of the CEC supported research project BREU P3091 "Assessment of Performance and Optimal Strategies for Inspection and Maintenance of Concrete Structures Using Reliability Based Expert Systems". The partners in the project are CSR, Aalborg, Denmark; University of Aberdeen, Aberdeen, UK/Sheffield Hallam University, Sheffield, UK; Jahn Ingenieurbureau, Hellevoetsluis, Holland; Instituto Superior Técnico, Lisboa, Portugal, and LABEIN, Bilbao, Spain

\section{REFERENCES}

1. Aitchison, J. \& I.R. Dunsmore. Statistical Prediction Analysis, Cambridge University Press, Cambridge, 1975.

2. Lindley, D.V. Introduction to Probability and Statistics from a Bayesian Viewpoint, Vol. 1+2, Cambridge University Press, Cambridge, 1976.

3. Madsen, H.O. Model Updating In Reliability Theory, Proc. ICASP5, pp. 564--577, 1987.

4. Rackwitz, R. \& K. Schrupp . Quality Control, Proof Testing and Structural Reliability, Structural Safety, Vol. 2, pp. 239--244, 1985.

5. Thoft-Christensen, P. \& M.J. Baker. Structural Reliability Theory and Its Applications, Springer Verlag, 1982.

6. Thoft-Christensen, P. \& Y. Murotsu . Application of Structural Systems Reliability Theory, Springer Verlag, 1986.

7. Thoft-Christensen, P. \& H.I. Hansen. Optimal Strategy for Maintenance of Concrete Bridges Using Expert Systems. Proc. ICOSSAR '93, Innsbruck, August 1993.

8. Thoft-Christensen, P. Assessment of Performance and Optimal Strategies for Inspection and Maintenance of Concrete Structures Using Reliability Based Expert Systems . Proc. ESReDA seminar on "Maintenance and System Effectiveness", Charmonix, France, April 1994, pp. 97-112. 